\title{
Design and commissioning of a high magnetic field muon spin relaxation spectrometer at the ISIS pulsed neutron and muon source
}

\author{
J. S. Lord, ${ }^{1}$ I. McKenzie, ${ }^{1}$ P. J. Baker, ${ }^{1}$ S. J. Blundell, ${ }^{2}$ S. P. Cottrell, ${ }^{1}$ S. R. Giblin, ${ }^{1}$ \\ J. Good, ${ }^{3}$ A. D. Hillier, ${ }^{1}$ B. H. Holsman, ${ }^{1}$ P. J. C. King, ${ }^{1}$ T. Lancaster, ${ }^{2}$ R. Mitchell, ${ }^{3}$ \\ J. B. Nightingale, ${ }^{1}$ M. Owczarkowski, ${ }^{3}$ S. Poli, ${ }^{3}$ F. L. Pratt, ${ }^{1}$ N. J. Rhodes, ${ }^{1}$ \\ R. Scheuermann, ${ }^{4}$ and Z. Salman ${ }^{1,2, a)}$ \\ ${ }^{1}$ ISIS Facility, STFC Rutherford Appleton Laboratory, Chilton, Oxon OX11 OQX, United Kingdom \\ ${ }^{2}$ Clarendon Laboratory, Department of Physics, Oxford University, Parks Road, Oxford OX1 3PU, \\ United Kingdom \\ ${ }^{3}$ Cryogenic Limited, 30 Acton Park Industrial Estate, The Vale, Acton, London W3 7QE, United Kingdom \\ ${ }^{4}$ Laboratory for Muon Spin Spectroscopy, Paul Scherrer Institut, CH-5232 Villigen PSI, Switzerland
}

(Received 11 April 2011; accepted 15 June 2011; published online 15 July 2011)

\begin{abstract}
The high magnetic field ( $\mathrm{HiFi}$ ) muon instrument at the ISIS pulsed neutron and muon source is a state-of-the-art spectrometer designed to provide applied magnetic fields up to $5 \mathrm{~T}$ for muon studies of condensed matter and molecular systems. The spectrometer is optimised for time-differential muon spin relaxation studies at a pulsed muon source. We describe the challenges involved in its design and construction, detailing, in particular, the magnet and detector performance. Commissioning experiments have been conducted and the results are presented to demonstrate the scientific capabilities of the new instrument. (O) 2011 American Institute of Physics. [doi:10.1063/1.3608114]
\end{abstract}

\section{INTRODUCTION}

The muon spin relaxation technique is used to study atomic-level properties of condensed matter and molecular systems. ${ }^{1-3}$ Spin polarized positive muons with an energy of $\approx 4 \mathrm{MeV}$ are implanted into a sample. The muons decay with a mean lifetime of $\approx 2.2 \mu$ s and emit a positron preferentially in the direction of their spin at the instant of decay. The evolution with time of the muon spin direction can be followed by calculating the asymmetry in the positron counting rate of detectors on opposite sides of the sample. The technique has applications in numerous areas that include investigation of spin dynamics and ordering in magnetic systems, superconductivity, diffusion, chemical radicals and reactions, and hydrogen behaviour in semiconductors or proton conductors.

The ISIS muon facility at the Rutherford Appleton Laboratory has been in its current form since 1994, when European funding provided for its expansion from one to three experimental areas. ${ }^{4}$ Protons from the ISIS synchrotron generate pulses of muons, of duration $80 \mathrm{~ns}$ every $20 \mathrm{~ms}$. As a pulsed source ISIS has particular strengths for muon studies, including a very low background count rate (uncorrelated with muon decays) which enables the evolution of the muon polarization to be followed out to at least 10 muon lifetimes. This makes ISIS suitable for measurements of very weak spin relaxation, or where structure exists in the polarization signal at long times. A pulsed source also allows efficient use of synchronous sample excitation, such as RF resonance.

Instruments providing fields of up to $\approx 7 \mathrm{~T}$ have been available for some time at the Paul Scherrer Institut (PSI), Switzerland (Ref. 5) and TRIUMF (Vancouver, Canada) (Ref. 6) muon sources. In general, these have been optimised

a) Present address: Laboratory for Muon Spin Spectroscopy, Paul Scherrer Institut, CH-5232 Villigen PSI, Switzerland. for experiments that can exploit the unique properties of the muon beam available at these facilities. Very high timing resolution is possible, but with limited data rates, achieved by correlating single arrival and decay events. Alternatively, if information on the muon polarisation evolution with time is discarded, time-integral data can be collected at high rates. Such instruments require small numbers of detectors located very close to the sample position.

In contrast, instrumentation at pulsed sources requires detector arrays with many elements to cope with the large instantaneous rates that follow implantation of the pulse of muons. This provides unique challenges for instrument design. However, the benefit is the provision of very high data rates while retaining the time evolution of the muon polarisation ("time-differential" measurements). An instrument providing time-differential measurement in fields up to $3.7 \mathrm{~T}$, optimised for radio frequency resonance measurements, has been constructed in the past at the pulsed muon facility at KEK (High Energy Accelerator Research Organization, Tsukuba, Japan). ${ }^{7}$ The design of the $5 \mathrm{~T}$ instrument at ISIS focused on providing a spectrometer complementary to those at continuous sources, designed specifically for time differential measurements with the applied field parallel to the initial spin direction ("longitudinal field") and capable of measuring weak signal decays at high data rates. This unique ability opens up new science areas to $\mu \mathrm{SR}:^{8,9}$ for example, the decoupling of fluctuating spins, the measurement of spin dynamics, and the exploration of magnetic phase diagrams are subjects where weak relaxation rates are frequently encountered. Spectroscopic techniques such as those which exploit level crossing resonances of radicals formed by muon implantation in organic systems and radio frequency methods also benefit from the inherent time differential data acquisition as relaxation data can readily be extracted from the signals. 


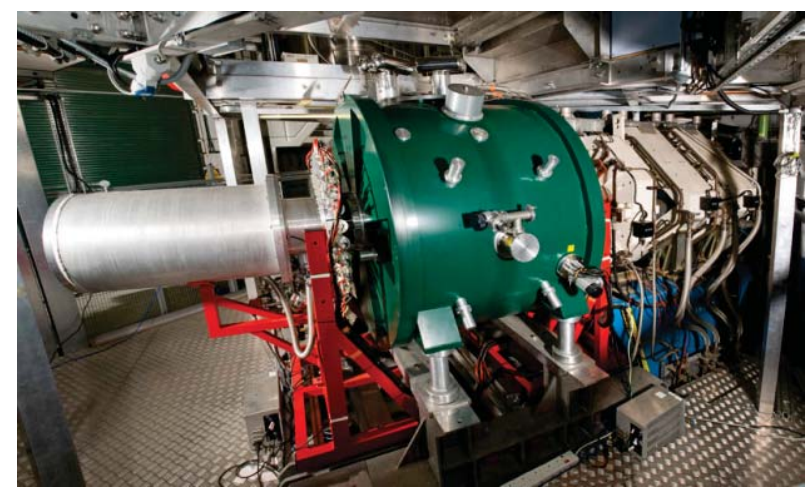

FIG. 1. (Color online) Photograph of the HiFi instrument showing (from right to left) the final quadrupole triplet, the superconducting magnet (large cylinder), the downstream detector bank and the flypast tank.

This paper describes the design and commissioning of the spectrometer (Fig. 1), considering the unique problems associated with measuring and analysing artefact-free time differential data to long times after muon implantation. Section II gives a detailed description of the instrument magnet, a oneoff, cryogen-free superconducting split-pair generating fields up to $5 \mathrm{~T}$, which needs a large bore, good homogeneity, high stability, and low external stray field. Section III describes the optimisation of muon transport through the region of varying field to the sample position at the centre of the magnet. Section IV considers the instrument detector array, designed with the aid of simulations to maintain a good count rate and data quality in all fields while avoiding saturation of any element with too high a count rate. Section V considers detector performance in practice. Sections VI and VII deal with data acquisition and sample environment. Finally, Sec. VIII presents a number of science examples in areas of physics and chemistry that demonstrate the unique capabilities of the new instrument.

\section{MAGNET}

The science case for the new instrument required magnetic fields up to at least $4 \mathrm{~T}$ and preferably $5 \mathrm{~T}$. This necessitated a superconducting magnet since the field range was too high for practical resistive magnets, even with iron cores. Many experiments need to scan a range of fields including complementary measurements in zero magnetic field (below $0.1 \mathrm{mT})$.

\section{A. Magnet specification}

A magnet satisfying our scientific requirements and giving the performance below was designed and built by Cryogenic Ltd, London, UK. The magnet is a cryo-free system, using pulse tube coolers ${ }^{10}$ which have no cold moving parts and produce very little vibration. Cool-down time of the magnet coils from room temperature is about 1 week.

The incoming muon beam is horizontal and the magnet's main field axis $(z)$ is parallel to this. Other axes are defined with $x$ horizontal and perpendicular to the beam, and $y$ vertical.
To allow vertical access to the sample position, permitting conventional liquid helium cryostats and a dilution refrigerator insert to be used, a split pair magnet design was preferred over a solenoid. An additional advantage of not having the sample environment equipment entering along the field axis is that "flypast" operation ${ }^{11,12}$ can be used, where muons not stopping in the sample are allowed to exit the spectrometer and their decay positrons are not counted. The drawback of a split pair is a more complex magnet design, making it harder to obtain the required homogeneity.

The magnet has a warm bore, allowing independent operation of the magnet with any cryostat or furnace. The bore is usually evacuated with cryostats using this as their insulating vacuum - this avoids the need for an outer room temperature cryostat "tail" and its associated window in the beam path (two windows in flypast operation) while maximising the volume available for the sample. This does, however, remove the ability to pre-cool cryostats off-line for a quick experimental change-over.

The vacuum in the magnet bore is separated from the beamline vacuum by a $50 \mu \mathrm{m}$ Mylar window, and this also allows the option of having the magnet bore at atmospheric pressure, for complex sample environment equipment running near ambient temperature.

The magnet has a main bore diameter of $300 \mathrm{~mm}$, large enough to contain the positron detectors (Sec. IV) and the light guides leading to them, while also allowing unobstructed entry of the converging muon beam. Similarly, the split was designed to enable a top bore of $100 \mathrm{~mm}$ and three other ports (sides and bottom) of $89 \mathrm{~mm}$, dimensions chosen to permit mounting of cryostats providing an ISIS standard $40 \mathrm{~mm}$ square sample stage.

Most muon experiments will use a range of magnetic fields and the time spent changing the field should be minimised to make optimum use of the available beam time on the instrument. The ramp times for the magnet are about $10 \mathrm{~min}$ from 0 to $1 \mathrm{~T}$ and $90 \mathrm{~min}$ to reach the full field of $5 \mathrm{~T}$.

Some experiments, such as RF resonance, will perform measurements at one constant field (either long, high statistics runs or varying other parameters such as temperature). For these we have the option of persistent mode, with very low drift (not more than $1 \mathrm{ppm}$ over $12 \mathrm{~h}$ ). Other experiments, for example, scanning through level-crossing resonances, may need to change the field every few minutes so that the magnet can also run in continuously driven mode, with the power supply giving stability of better than $20 \mathrm{ppm}$ over $12 \mathrm{~h}$. To follow magnetic hysteresis loops and prepare metastable magnetically ordered states, the magnetic field is reversible.

Resonance experiments need good homogeneity of the field in order that a resonance line is not significantly broadened or precession signals damped. For a typical small solid sample (a disc of diameter $20 \mathrm{~mm}$ perpendicular to the field), the magnet provides homogeneity better than $20 \mathrm{ppm}$. For larger sample setups, such as gas cells, or to allow a sample to be rotated to align different crystal axes with the field, a homogeneity of $100 \mathrm{ppm}$ is provided over a larger volume ( $40 \mathrm{~mm}$ diameter by $40 \mathrm{~mm}$ long cylinder). 
Some measurements of level-crossing resonances use magnetic field modulation of amplitude comparable to the expected linewidth (usually 5-10 mT) to cancel out any gradual drifts in the beam or sample position or detector sensitivity. Subtracting the two average muon polarisations gives a differential lineshape. An additional $z$ coil is provided for this, and is arranged to have a zero mutual inductance with the main field coil, so that modulation is unaffected when the main coil is in a persistent state. The power supply can be controlled by the data acquisition electronics (Sec. VI) to switch the field automatically and collect two data sets for the two field values.

Calibration measurements (Sec. V C) need a small magnetic field in a transverse direction, and we often also need to measure in "zero" magnetic field to complement the higher field data. For these, we have additional coils to apply fields in the $x$ and $y$ directions, up to $10 \mathrm{mT}$ and reversible. The $z$ coil can also be used for zero field correction or setting low longitudinal fields. It has a maximum field of $40 \mathrm{mT}$ and is also reversible.

The magnet is located close to two other muon spectrometers. To ensure that any stray field does not deflect the muon beams leading to them, or alter the field on their sample positions, the magnet has a stray field of below $0.2 \mathrm{mT}$ at $3 \mathrm{~m}$ from the magnet centre. To achieve this, the magnet is self-shielded with extra coils to oppose the external stray field.

The whole instrument including the magnet is mounted on a slide assembly, allowing it to be moved along the beam axis for maintenance of the beamline window. The detector banks, which enter the magnet through the ends of the main bore, are on subsidiary slides, so they can be withdrawn from the magnet and then disconnected and lifted off, if clear access into the magnet bore is required.

\section{B. Correcting for remanent fields}

Any superconducting magnet will have a remanent field depending on the recent field history when the current is turned off, even with the persistent mode switch open. To measure and correct for this we fitted two groups of three perpendicular Hall probes. These are model MPT-141 single axis sensors with an extended calibration range, each with a DTM151 readout unit, from Group 3 Technology Ltd., Auckland, New Zealand. They are located behind the detector banks and away from the muon beam, at positions $(0,+y,+z)$ and $(0,+y,-z)$, while still close to the centre. The remanent field profile would be expected to have a constant component plus an axially symmetric quadratic variation with position: $\mathbf{B}=B_{1} \hat{\mathbf{z}}+B_{3}\left(\left(2 z^{2}-x^{2}-y^{2}\right) \hat{\mathbf{z}}-2 x z \hat{\mathbf{x}}-2 y z \hat{\mathbf{y}}\right)$. This is confirmed by measurements along the $x$ axis, which show that after an excursion to the maximum positive field, the field at the centre is about $+1.4 \mathrm{mT}$, and $+1.6 \mathrm{mT}$ in the central plane at a radius of $50 \mathrm{~mm}$. Subsequent drifts on the timescale of hours to days are negligible compared to external stray fields.

By comparing the two measured $B_{y}$ fields, we can calculate the magnitude of $B_{3}$ and thus the field $\mathbf{B}$ at the sample. To set a "zero" field, the $x, y$, and $z$ coils are used to oppose this ( $x$ and $y$ mainly cancel stray fields of external origin, while $z$ cancels the remanent field). When setting low transverse or longitudinal fields using the $x, y$, or $z$ coils, these offset values are added (a transverse field must be perpendicular to the muon spin to give an accurate calibration).

The Hall probe system was calibrated to correct for any slight misalignments of the sensors (probably below $2^{\circ}$ ), their exact positions within the magnet, field gradients resulting from the $x, y$, and $z$ coils themselves, and any constant external field gradients, for example, from the beamline quadrupole magnets. A triple axis fluxgate sensor was put at the sample position, then the main field was run up to various positive or negative values and back to zero, and the $x, y$, and $z$ coils were used to zero the field on the fluxgate probe. The three field settings and six Hall probe readings were then recorded. The resulting performance of the system routinely provides a zero field to within $0.01 \mathrm{mT}$ regardless of the previous magnetic field history.

\section{MUON BEAM TRANSPORT}

$\mathrm{HiFi}$ is located on an existing muon beamline. Due to the physical size of the magnet and space required for upstream detectors, and to reduce the force on the iron yoke of the final beamline quadrupole due to the magnet's fringe field, the beam is brought to a focus at $1 \mathrm{~m}$ from the downstream face of the final quadrupole. Remote collimation slits upstream of the quadrupoles are used to adjust the horizontal spot size and rate.

In order to focus and steer the spot, a field-insensitive beam camera was constructed, based on a scintillator screen mounted at the sample position and a high sensitivity cooled CCD camera (model SXVF-H9 from Starlight Xpress Ltd.) and lens viewing this from downstream. The scintillator is mounted on the sample stage for the closed-cycle refrigerator (CCR) (Sec. VII), so accurate alignment between the spot and the sample can be assured.

As predicted from simulations, ${ }^{13}$ the beam spot size and shape clearly varies with field (Figs. 2 and 3), as the spot is focused and defocused, and rotated around the field axis. However, the integrated intensity is constant, showing that the camera's sensitivity is unaffected, and no beam is lost in the upstream fringing fields. The form of the simulation shows a remarkable agreement with the measurement, while the absolute simulated spot size is smaller as the TURTLE (Ref. 14) model uses an idealised beam profile from the upstream beamline.

Around 2.5 and 4.0 $\mathrm{T}$ the beam is tightly focused on the sample (Fig. 3), so these are good fields to work at with small samples, if measurements are required at essentially constant field and this need not match any intrinsic resonance value. RF resonance, at a high frequency where it is beneficial to use a small coil, is an example of such an experiment.

The beam position was also found to vary with field as expected, since even small misalignment errors between the beam envelope and the field axis will cause the mean beam position to spiral round the field axis. The effect is largest over the field range $0-1 \mathrm{~T}$ where the spot was found to move round the spiral by $180^{\circ}$ or about $7 \mathrm{~mm}$, while in higher fields the spot converges on a point defined by the magnetic field 


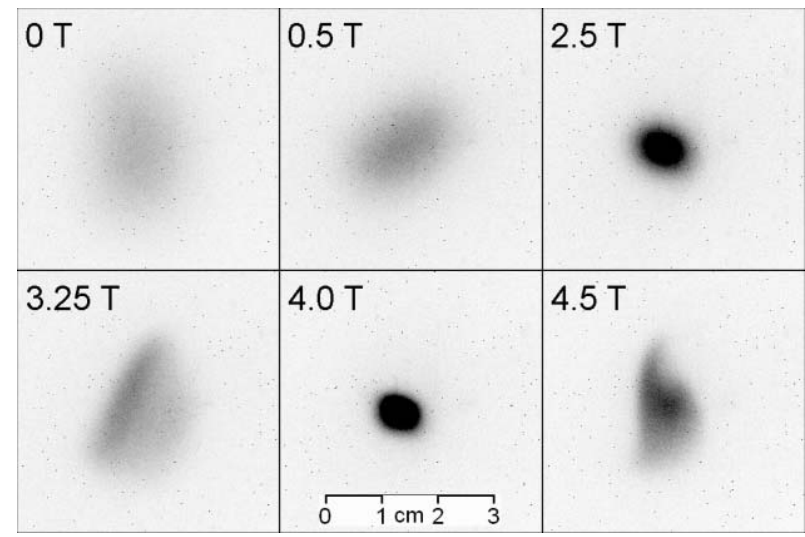

FIG. 2. Observed beam spot at the sample position, in fields of 0.0, 0.5, 2.5, $3.25,4.0,4.5 \mathrm{~T}$.

profile of the magnet. To correct for this beam movement a set of three steering magnets was installed upstream of the final quadrupole triplet, with the last bending magnet providing the final adjustment enabling independent positioning of beam position and angle in the $x$ and $y$ planes. By measuring steering curves in various fields a well-aligned setting can be achieved which limits the spot movement to less than $1 \mathrm{~mm}$.

For flypast operation, muons not stopping in a small sample continue through a hole in the backward detector bank, out of the magnet bore and into a large diameter extension to the downstream beam pipe, still in vacuum. At some fields the diverging beam from the sample position would be defocused further, and could cause muons to stop close to the detectors if the diameter was restricted to that of the incoming beam pipe.

The signal to background ratio was estimated by measuring total positron count rates with no sample present, with a flypast test sample, and with a large plate, shown in Fig. 4. In low fields some muons in the halo of the beam spot will stop in the cryostat tails or around the backward detectors, giving a background signal, since the beam converges towards the focus at the sample position and diverges beyond it. In high fields the beam converges in the fringing field before the upstream detectors and then oscillates between well

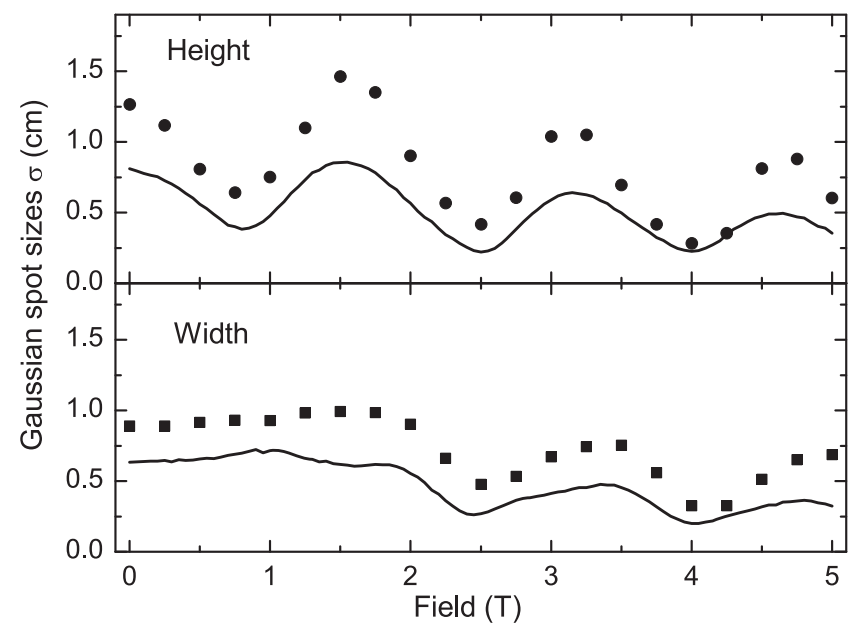

FIG. 3. Variation of the muon spot size with field (symbols) compared to the simulation (lines).

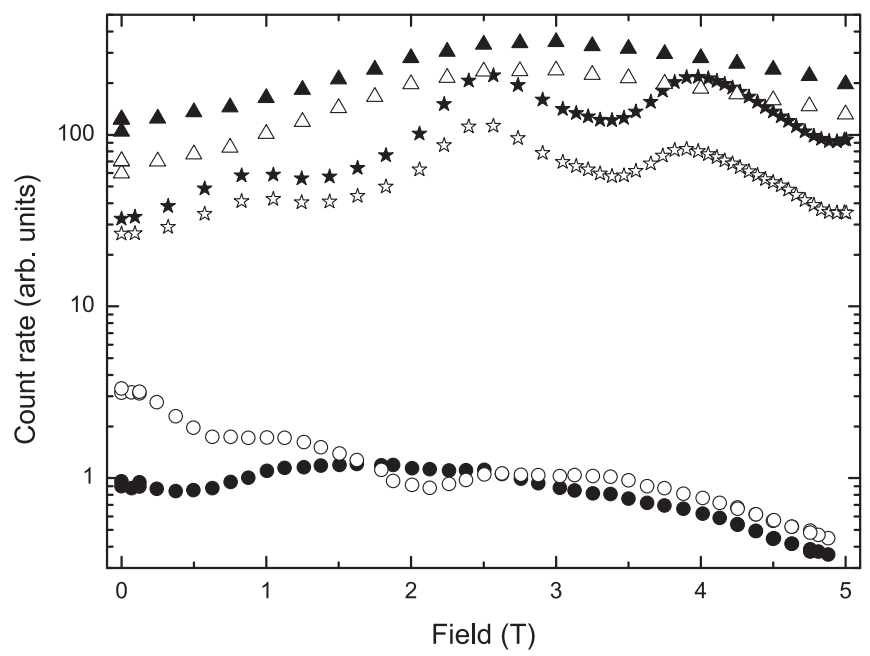

FIG. 4. Total count rates in the forward (filled symbols) and backward (open symbols) detector banks with field, for slits $=15 \mathrm{~mm}$. Circles: with the CCR (closed cycle refrigerator) radiation shield and windows but no sample. Stars: a $10 \times 10 \mathrm{~mm}$ Ag flypast sample, in the CCR. Triangles: a large Ag plate, slits $=8 \mathrm{~mm}$.

defined minimum and maximum radii while passing through the cryostat windows. The positron count rates with a 10 $\times 10 \mathrm{~mm}$ sample exceed the background rate by a factor of 10 in low field and 100 at $2.5 \mathrm{~T}$ and above.

\section{DETECTOR DESIGN}

The positrons emitted by muon decay have a distribution of energies up to $52.83 \mathrm{MeV}$ and will follow helical paths in a uniform magnetic field. We wished to maintain a high count rate in high fields, capturing a large fraction of those positrons which carry useful information on the muon polarisation (at the higher end of the energy spectrum, and nearly parallel or antiparallel to the muon spin rather than perpendicular).

The radius of the positron helix, for positrons emitted approximately perpendicular to the field and with maximum energy, is $3.5 \mathrm{~cm}$ in a field of $5 \mathrm{~T}$. The useful positrons for measurement of longitudinal polarisation, emitted within $45^{\circ}$ of

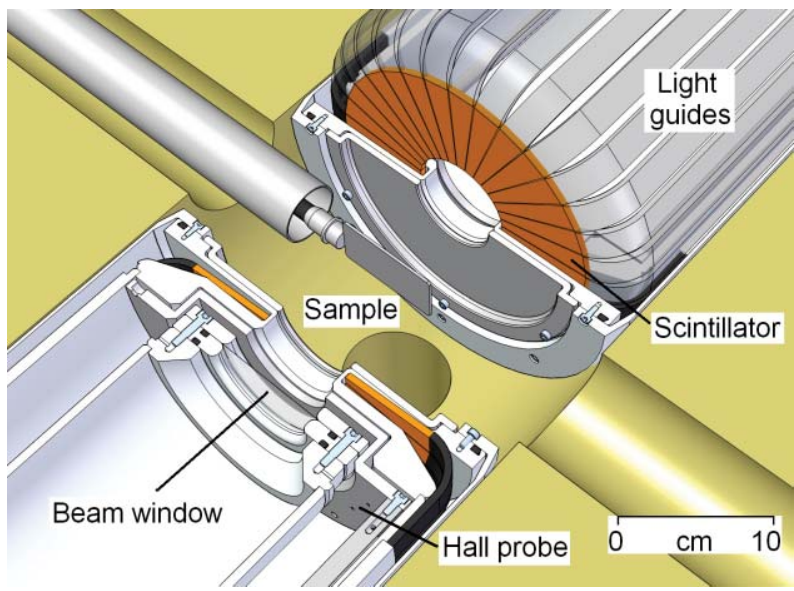

FIG. 5. (Color online) Cut-away diagram showing the arrangement of the detectors in their vacuum vessels around the sample position. The beam enters from the bottom left. 
the beam axis, will have a radius of up to $2.5 \mathrm{~cm}$, so they will not travel more than $5 \mathrm{~cm}$ transverse to the muon's stopping site. Therefore, detector elements need to be placed sufficiently close to the beam axis in order to be able to measure any data at all in the highest fields.

Similarly, in the longitudinal direction, the positrons emitted approximately parallel to the field, again with maximum energy, all have a helical pitch of about $2 \pi \times 3.5=22$ $\mathrm{cm}$, and are refocused onto the field axis at this distance. A detector intended to measure longitudinal polarisation from the near parallel positrons but which requires a small central hole for the incoming beam or the exiting "flypast" muons, could be located at the antinode, $11 \mathrm{~cm}$ from the sample. To detect positrons emitted at higher angles, and for a range of energies near the maximum, the detector should be placed slightly nearer to the sample.

Analysis of longitudinal muon data usually uses the asymmetry $a(t)$ (Eq. (1)) with a calibration factor $\alpha$ representing the ratio of efficiencies and solid angles of forward and backward detectors so that $a(t)=0$ when the muons are fully depolarised. The array is a symmetrical arrangement of forward and backward detector banks so that, with an ideal sample, the calibration factor $\alpha$ (Sec. V C) should be 1 at all fields. Such a sample would be centrally located and has thickness (including any backing or holder) equal to twice the muon range, so the positrons exiting through front or back faces are degraded by the same amount.

To handle high data rates, the detector banks must be segmented, with a geometry designed to minimise "double counting" (Sec. V B) when a high energy positron passes through more than one element and gives a count in each. A flat plate detector geometry, perpendicular to the field, has the advantage that any helical track only intersects it once, and any double counting is limited to tracks crossing the gap between adjacent elements.

Each circular detector plate is subdivided into sectors. With too many elements the double counting fractions in high fields would increase and eventually the count rate per detector no longer decreases however many more elements are added. A very highly segmented detector has short path lengths (especially at high field) in the scintillator elements, reducing light output and signal-to-noise ratio. There is also more lost solid angle from inter-element gaps. The optimum for a detector of this overall size is around 32 elements per bank. The detectors are not subdivided into rings of different radii, since at high fields only the inner ring would contribute any counts at all and the total count rate able to be handled would not increase. The detector elements could have been angled in the manner of a turbine blade, with the gaps parallel to the high field positron tracks, to reduce double counting. As this would only work for one sign of the magnetic field and increase double counting for the other sign and in low fields, we chose to preserve symmetry between field directions by having straight edged elements.

The detectors (Figure 5) comprise BC408 plastic scintillator $(5 \mathrm{~mm}$ thick) connected at the outer edge to light guides of $5 \times 20 \mathrm{~mm}$ cross section, which curve with a radius of $30 \mathrm{~mm}$ to exit along the main bore and then to pho- tomultiplier tubes (PMTs) located outside. The scintillators and light guides are in air, in a re-entrant vacuum vessel, to avoid the need for optical feedthroughs. The field-tolerant PMTs (Hamamatsu R5505) are oriented radially with respect to the magnet axis, at a point where the fringing field is also radial (parallel to the tube axis, its least sensitive direction). The stray field here has a magnitude of $\sim 0.15 \mathrm{~T}$ with a main field of $5 \mathrm{~T}$, so little variation of gain with field was expected or measured. No mu-metal shields are used because the fields are too high to be shielded completely.

To simplify assembly of the detector bank, after wrapping the scintillators and light guides in foil, they were assembled in groups of four, and the light guides were bonded together with an epoxy resin to ensure that they are light-tight. Eight such modules were arranged round the inner part of the detector housing on each side of the instrument.

\section{DETECTOR PERFORMANCE}

\section{A. Thresholds, count rates, and dead times}

High voltage (HV) is provided by a CAEN model SY1527 power supply with model A1535SP output modules. The PMTs were run with a positive HV, with the photocathode near the ground potential. Pulses are detected via CAEN V895 discriminator modules, programmable via a Versa Module Europa (VME) crate.

Pulse height spectra can be measured in various applied fields and the mean value is found to change relatively little though the distribution broadens. Dead times, the time after one event before another can be counted in the same detector element, are calibrated in the conventional manner, with typical values of $10 \mathrm{~ns}$, and also vary relatively little with field.

The overall count rate varies with field, generally increasing from low field to a peak around 2-3 T and then falling off at higher fields, as in Fig. 4. This variation depends to some extent on the degrading effect of the sample and the holder, and cryostat tails. The numbers could be represented as an approximate solid angle though this is an average over positron energies rather than a clearly defined angular range (the geometric solid angle covered by detectors being $0.33 \times 4 \pi \mathrm{sr}$ ). The variation must be allowed when setting up the beam slits for an experiment that will scan a wide field range.

\section{B. Double counting}

Double counts occur when one positron passes through two detector elements and gives a signal above threshold in each. This effect can be measured "in situ," from any data set, by looking for increased correlations in the random noise between adjacent detector elements. We can calculate a correlation value,

$$
C_{\alpha, \beta}(\Delta t)=C_{\alpha, \beta}(j \delta t)=\frac{\sum_{i=n_{1}}^{n_{2}} N_{\alpha}(i) N_{\beta}(i+j)}{\left[\sum N_{\alpha}(i) \times \sum N_{\beta}(i)\right]},
$$

where the sum over bins $n_{1}$ to $n_{2}$ of width $\delta t$ is towards the end of the histograms where count rates, and accidental correlations between two events in the same time bin, are lower. 
The auto-correlation $C_{\alpha, \alpha}$ shows a sharp peak at $\Delta t=0$. Between adjacent elements, $C_{\alpha, \alpha+1}$ shows a smaller peak (of finite width, due to the time resolution of the detector chains) if there are any double counting events common to both histograms.

An absolute estimate of double counting fraction can be made by comparing the areas of the auto-correlation and cross-correlation peaks (for data taken with the usual bin widths of $16 \mathrm{~ns}$, only $\Delta t=0$ and $\Delta t \neq 0$ points need to be calculated). It is found to increase linearly with field as was predicted in simulations, ${ }^{9}$ reaching about $25 \%$ at $5 \mathrm{~T}$, see Fig. 6. On this scale the pure double counting case (each event in two adjacent histograms) would be $50 \%$ and the limiting value of $100 \%$ means all events are present in both histograms - each event being counted many times in a group of detector elements. For improved statistics we can calculate the individual correlation functions and then average them over all detectors, or adjacent pairs, in one bank-or over several low statistics runs.

Since these double counts correspond to "good" positrons carrying useful polarisation information, no improvement can be made to the resulting data quality if there were some way to veto them. The consequence is an increase in the $\chi^{2}$ for a least-squares fit of the measured data, when the histograms are grouped to bring together the double counts, by a factor of 1.5 at $5 \mathrm{~T}$. The errors on fit parameters are also slightly reduced, to 0.8 of the true value at $5 \mathrm{~T}$.

The time resolution estimated from double-counting correlation is about $1.4 \mathrm{~ns}$ giving a frequency response falling to half amplitude at $190 \mathrm{MHz}$, only relevant or observable on the ISIS beam when data acquisition is synchronised to a pulsed stimulus, such as RF. Including all the positrons which are not double-counted may change this figure, as might the spiralling of the positrons from the sample to the first detector element reached. However, some improvement may be made by using constant-fraction discriminators.

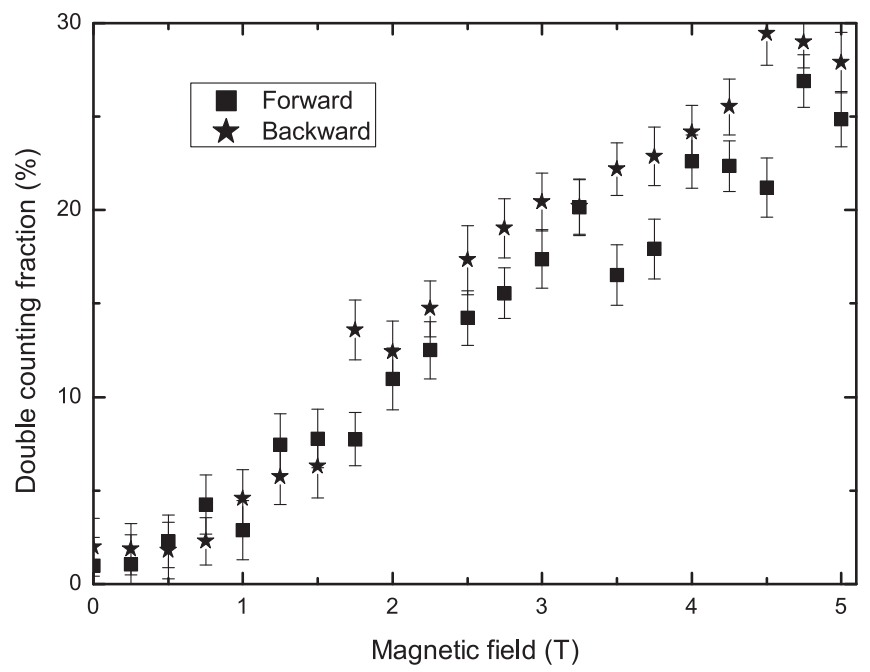

FIG. 6. Double counting fraction estimate as a function of field. Sample: $125 \mu \mathrm{m} \mathrm{Ag}$ sheet.

\section{Data reduction}

Muon spin relaxation data is usually grouped into total forward and backward count rates $F(H, t)$ and $B(H, t)$ (where $t$ is the time after muon implantation) and is then reduced to obtain the forward-backward asymmetry $a(H, t)$ of positron decays, and depolarization function $G_{z}(H, t)$, where:

$$
a(H, t)=a_{1}(H) G_{z}(H, t)=\frac{F(H, t)-\alpha(H) B(H, t)}{F(H, t)+\alpha(H) B(H, t)} .
$$

Alternatively, the detectors or groups can be analysed individually by compensating for the muon lifetime $\tau_{\mu}$ :

$$
\begin{aligned}
F(H, t) & =N_{0 F} \exp \left(-t / \tau_{\mu}\right)(1+a(H, t)) \\
& =N_{0 F} \exp \left(-t / \tau_{\mu}\right)\left(1+a_{1 F} G_{z}(H, t)\right) .
\end{aligned}
$$

Usually a low transverse field (2-10 mT) is applied and the calibration factor $\alpha$ in Eq. (1) (allowing for relative detector efficiency) is adjusted so that $a(t)$ oscillates about zero. $\alpha$ is a function of the sample thickness and its exact position within the spectrometer.

One problem in high field muon spectrometers is that both $\alpha$ and maximum relaxing asymmetry $a_{1}$ vary as a function of field due to positron spiralling. If not allowed for, this will effectively give an offset and/or scale factor to the extracted $G_{z}(H, t)$.

A useful calibration can be made with a sample showing full initial polarisation and relaxing on a measurable timescale $\lambda \approx 0.5-1 \mu \mathrm{s}^{-1}$ to zero at all fields. It is important that the relaxation is a simple exponential (or other known form) and all muons take part. One such example is a semiconductor at a high enough temperature such that charge cycling occurs, around $400 \mathrm{~K}$ for germanium or higher for silicon. Some paramagnetic materials may also be used. Given an expected relaxation $G_{z}(H, t)=\exp (-\lambda(H) t)$, data taken at any field $H$ can be fitted to Eq. (1) to obtain $\alpha(H)$ and an average asymmetry $a_{1}(H)$ (and $\lambda(H)$ which is usually also a function of temperature). In addition, the individual groups can be fitted to Eq. (2) to obtain the relaxing asymmetry $a_{1 F}$ or $a_{1 B}$ seen by that group.

Various sample configurations were tried, and they show that $\alpha$ may vary with field and also depend on the sample thickness. The relaxing asymmetry $a_{1}$ has a fairly reproducible field variation, Fig. 7. The asymmetry in forward detectors (in the direction of the initial muon spin, with muons entering and positrons exiting the same face of the sample) does not vary with the sample setup. The exception is flypast in low field where some muons may stop in the cryostat walls, where their spins do not relax and, on average, they are nearer the backward detector bank. In the backward direction the increased degrading effect of a thick sample holder results in an increase in asymmetry, and reduction in rate as revealed by $\alpha>1$. This is more significant in higher field where the resulting lower energy positrons may not be able to reach the detectors. As expected a thin, symmetrical sample gives the least $\alpha$ variation.

If there is some temperature where a sample being studied is known to give full initial asymmetry at all fields, it can be used to calibrate $\alpha$ versus field for that sample, by 


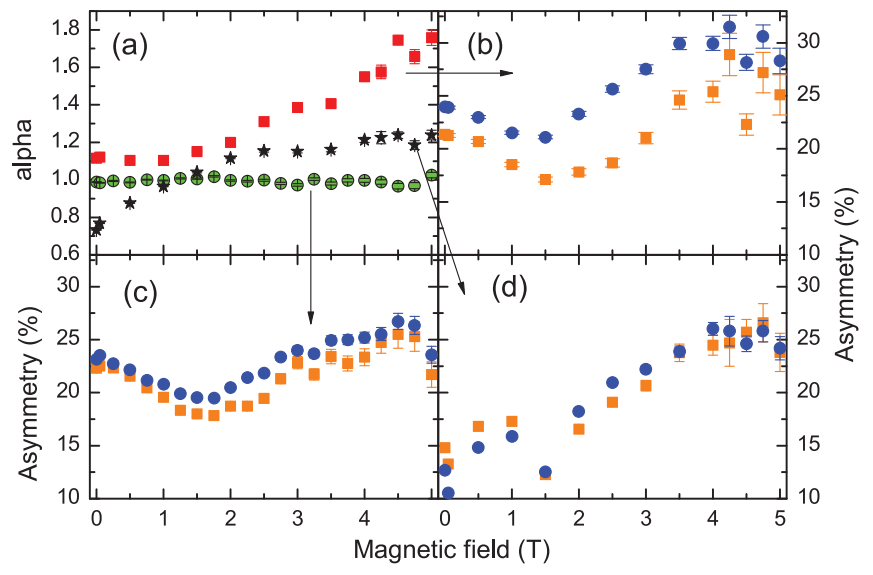

FIG. 7. (Color online) (a) Variation of alpha (Eq. (1)) with field, measured with germanium at $400 \mathrm{~K}$. Squares: Ge wafer $(400 \mu \mathrm{m})$ on the standard $3 \mathrm{~mm}$ thick aluminium sample blade of the flow cryostat. Circles: Ge wafer suspended in a thin aluminium foil packet. Stars: Flypast setup, small piece of Ge wafer $(5 \times 10 \mathrm{~mm})$ suspended in $\mathrm{Al}$ foil. (b), (c), (d) Variation of relaxing asymmetry (Eq. (2)) in the forward (squares) and backward (circles) detector banks, for the same three setups.

assuming a standard form for $a_{1}(H)$. Alternatively, a matching dummy sample of the same mass and area could be used for calibration.

These calibrations were performed with large samples to cover the whole beam, apart from the flypast case. On smaller samples mounted on a silver backing plate or with a mask, the variation of the spot size with field will change the fraction of the beam incident on the sample.

\section{DATA ACQUISITION}

HiFi uses the ISIS standard DAE-2 acquisition system with fast time-to-digital conversion (TDC) cards based on the Lecroy MTD 133b TDC chips. The time bin resolution can be set down to $500 \mathrm{ps}$ although for most relaxation measurements where the muon pulse length of $\approx 80 \mathrm{~ns}$ limits the frequency resolution, $16 \mathrm{~ns}$ bins are sufficient. Histograms of positron counts in each detector are generally collected to $32 \mu$ s after muon implantation. A Cerenkov counter picking up higher energy radiation from the muon production target is used as a start signal for the TDC, unless the data acquisition must be synchronised to a RF waveform or other sample stimulus. A controller program, named SECI, has been developed at the ISIS facility for experimental control of muon and neutron instruments, and is used to control the HiFi instrument. National Instruments LABVIEW drivers are used for the magnetic field control and various sample environment equipment.

\section{SAMPLE ENVIRONMENT EQUIPMENT}

The equipment provided initially is:

- CCR, with temperature range from 4 to $400 \mathrm{~K}$. The sample is on a cold finger in vacuum, up to $50 \mathrm{~mm}$ square allowing space for sealed cells with clamped windows.
- Flow cryostat, with temperature range $4-400 \mathrm{~K}$. The sample is in exchange gas, up to $40 \mathrm{~mm}$ square.

- Dilution refrigerator, with temperature range $30 \mathrm{mK}-$ $6 \mathrm{~K}$ in dilution mode, using a weak thermal link between the mixing chamber and the sample. The insert can also be operated up to $300 \mathrm{~K}$ with the mixture removed. Samples are $40 \mathrm{~mm}$ square on a cold finger. The magnet ramp rate can be reduced to essentially eliminate eddy current heating.

- Furnace. The sample is suspended in vacuum and heated by two IR lamps. ${ }^{15}$ The sample can be up to $40 \mathrm{~mm}$ square. Temperatures up to $1500 \mathrm{~K}$ can be achieved. Flypast operation is always used.

\section{EXPERIMENTS}

The HiFi spectrometer is now a part of the ISIS user programme. Experiments so far have used solid samples (in various cells and holders), liquids (in sealed cells), condensed gases, and RF excitation. Some examples are given here that cover topics in both physics and chemistry and demonstrate the application of RF excitation.

\section{A. Magnetic decoupling in $\mathrm{Ca}_{3} \mathrm{Co}_{2} \mathrm{O}_{6}$}

To demonstrate the effectiveness of HiFi in studying the decoupling of magnetic fluctuations in strongly magnetic systems, the muon spin relaxation rate in $\mathrm{Ca}_{3} \mathrm{Co}_{2} \mathrm{O}_{6}$ was measured as a function of field at $15 \mathrm{~K}$. This compound is a frustrated chain magnet which exhibits a partial magnetization plateau between 0.5 and $3.5 \mathrm{~T}$ in its intermediate temperature phase. ${ }^{16,17}$ Data recorded at different fields are shown in Fig. 8(a). All data could be fitted using a single exponential relaxation function, $A \exp (-\lambda t)$, and the resulting $\lambda$ values are shown in Fig. 8(b). Since the data take an exponential form over the measured field range we analysed the field-dependence of the $\lambda$ values using Redfield's equation: ${ }^{18}$

$$
\lambda=\frac{2 \gamma_{\mu}^{2} \Delta^{2} \tau}{1+\gamma_{\mu}^{2} \mu_{0}^{2} H^{2} \tau^{2}},
$$

where $\gamma_{\mu}$ is the muon's gyromagnetic ratio, $\Delta$ is the width of the local magnetic field distribution at the muon site, $\tau$ is the characteristic fluctuation time in the system, and $H$ is the applied longitudinal field. Reference 17 performs this analysis on $\mathrm{Ca}_{3} \mathrm{Co}_{2} \mathrm{O}_{6}$ at $20 \mathrm{~K}$ for the low field region, up to $0.4 \mathrm{~T}$, which we are able to extend to higher field. Three regions are observed as a function of field: from zero to around $0.5 \mathrm{~T}$ where the magnetization is increasing rapidly, from 0.5 to $3.6 \mathrm{~T}$ in the partial magnetization plateau, and above $3.6 \mathrm{~T}$ where the relaxation rate is approximately independent of the field. Fitting with Eq. (3) in the plateau region allows us to estimate the distribution of local fields at the muon site to be $\Delta \sim 40 \mathrm{mT}$ and the fluctuation timescale $\tau \sim 880 \mathrm{ps}$. This is consistent with the anticipated partially disordered antiferromagnetic state. ${ }^{16,17}$ 

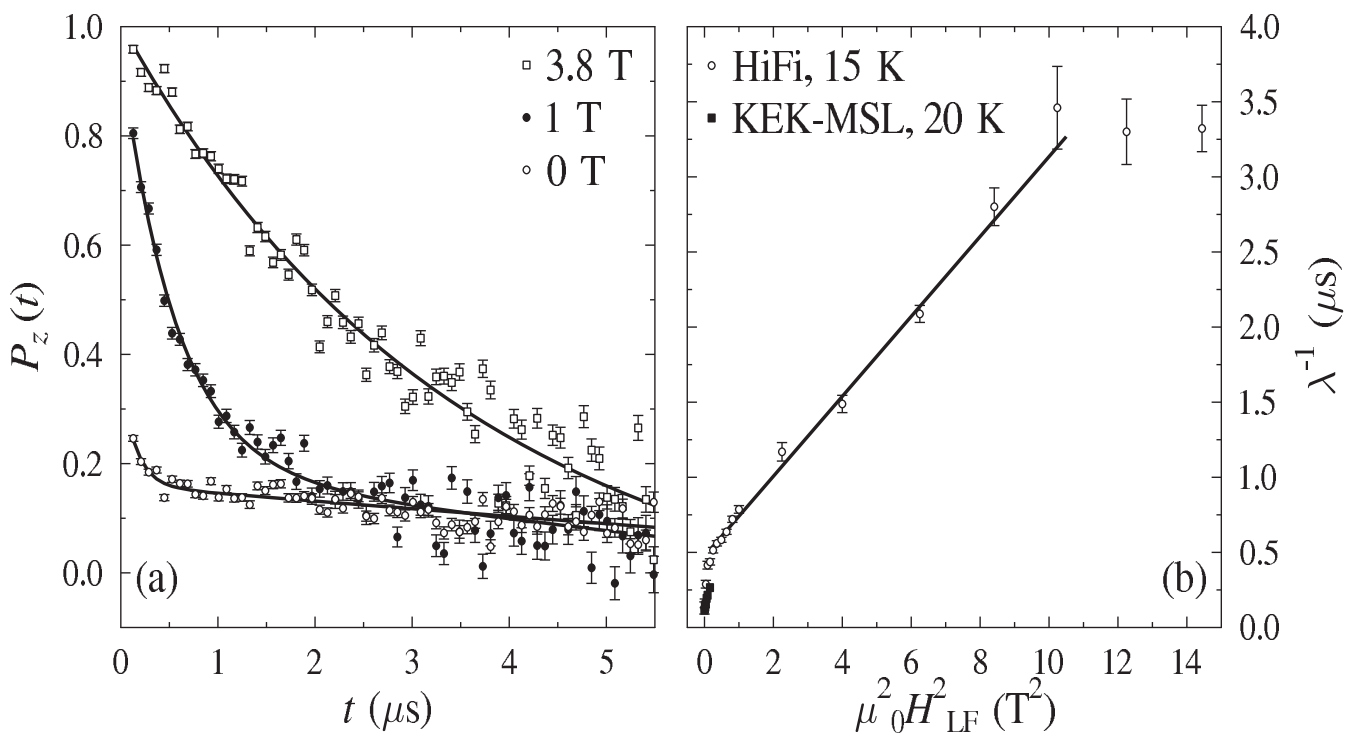

FIG. 8. (a) Asymmetry data recorded at $15 \mathrm{~K}$ in different applied fields. (b) Plot of $\lambda^{-1}$ against $H^{2}$ with a fit to Eq. (3) in the magnetization plateau region. Data from Fig. 6 of Ref. 17 included for comparison.

\section{B. Avoided level crossing muon spin resonance of the mu adducts of norbornene}

Radicals can be formed by the reaction of muonium, a light hydrogen isotope, with unsaturated bonds. The structure and dynamics of a radical can be determined by measuring the magnitude and temperature dependence of the hyperfine coupling constants (hfccs). The hfccs can be measured by the avoided level crossing muon spin resonance (ALC- $\mu \mathrm{SR}$ ) technique. ${ }^{19,20}$ The isolated spin states in high magnetic fields are essentially the products of the unpaired muon, electron, and nuclear Zeeman states, so there is no evolution of the muon spin with time and the asymmetry is independent of the applied field. At specific values of the applied field, nearly degenerate pairs of spin states can be mixed through the isotropic and anisotropic components of the hyperfine interaction. The muon polarization oscillates between the two mixing states of the avoided crossing. This results in a resonant-like change in the asymmetry as the magnetic field is swept. There are three types of resonances, which are characterized by the selection rule $|\Delta M|=0,1$, and 2 , where $M$ is the sum of the quantum numbers for the $z$ components of the muon and nuclear spins. The resonance field $\left(B_{\text {res }}\right)$ is given to first order by

$$
B_{\mathrm{res}}=\frac{1}{2}\left|\frac{A_{\mu}+(|\Delta M|-1) A_{X}}{\gamma_{\mu}+(|\Delta M|-1) \gamma_{X}}\right|,
$$

where $A_{\mu}$ is the muon hfcc, $A_{X}$ is the nuclear hfcc, $\gamma_{\mu}$ is the muon gyromagnetic ratio, and $\gamma_{X}$ is the nuclear gyromagnetic ratio. ${ }^{19}$

Norbornene (bicyclo[2.2.1]hept-2-ene) is a near-globular molecule and it exhibits a plastic solid phase with fast rotations of the molecules. Mu adds to the double bond of norbornene to give the radicals shown in Fig. 9. ${ }^{19,21}$ The $|\Delta M|=$ 1 resonance of the "exo" adduct of norbornene was observed at room temperature in both time-integral mode (Fig. 10(a)) and as a peak in the relaxation rate (Fig. 10(b)), although this requires substantially higher statistics per field point.

\section{RF resonance}

Application of a RF magnetic field can flip the muon spin if the frequency matches the splitting of the muon's allowed energy levels. In high magnetic fields, resonant frequencies will be centred on the Zeeman splitting $f=\gamma_{\mu} B / 2 \pi$ which reaches $677 \mathrm{MHz}$ at $5 \mathrm{~T}$. The muon's spin is largely decoupled from other spins by the field, indicating that a RF field intensity $B_{1}$ of $0.5-2 \mathrm{mT}$ is appropriate since it is acting on the muon's magnetic moment alone.

The high frequency requires a small coil with the tuning and matching capacitors located near it (well within a quarter wavelength, at $677 \mathrm{MHz} \lambda / 4=11 \mathrm{~cm}$ ). High frequencies also imply larger peak voltages across the coil for the same $B_{1}$ field, so the risk of electrical breakdown is greater.

We can usually choose a field for resonance measurements where the spot size is at a minimum (Sec. III), so that a small sample can be used, and a smaller, lower inductance coil makes high frequency operation easier. Flypast operation is to be preferred; any muons stopping in the metal cryostat walls do not see the RF field, since the electrical skin depth is much smaller than the muon stopping range, and a silver
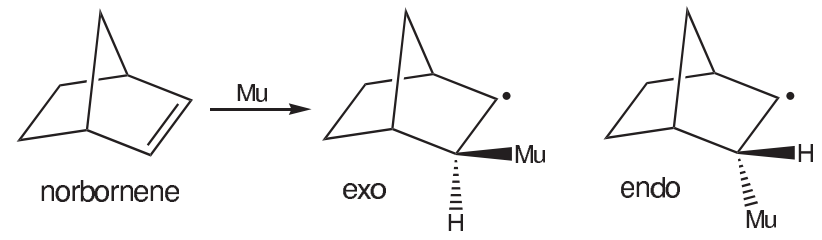

FIG. 9. Structures of norbornene and the two types of Mu adducts. 


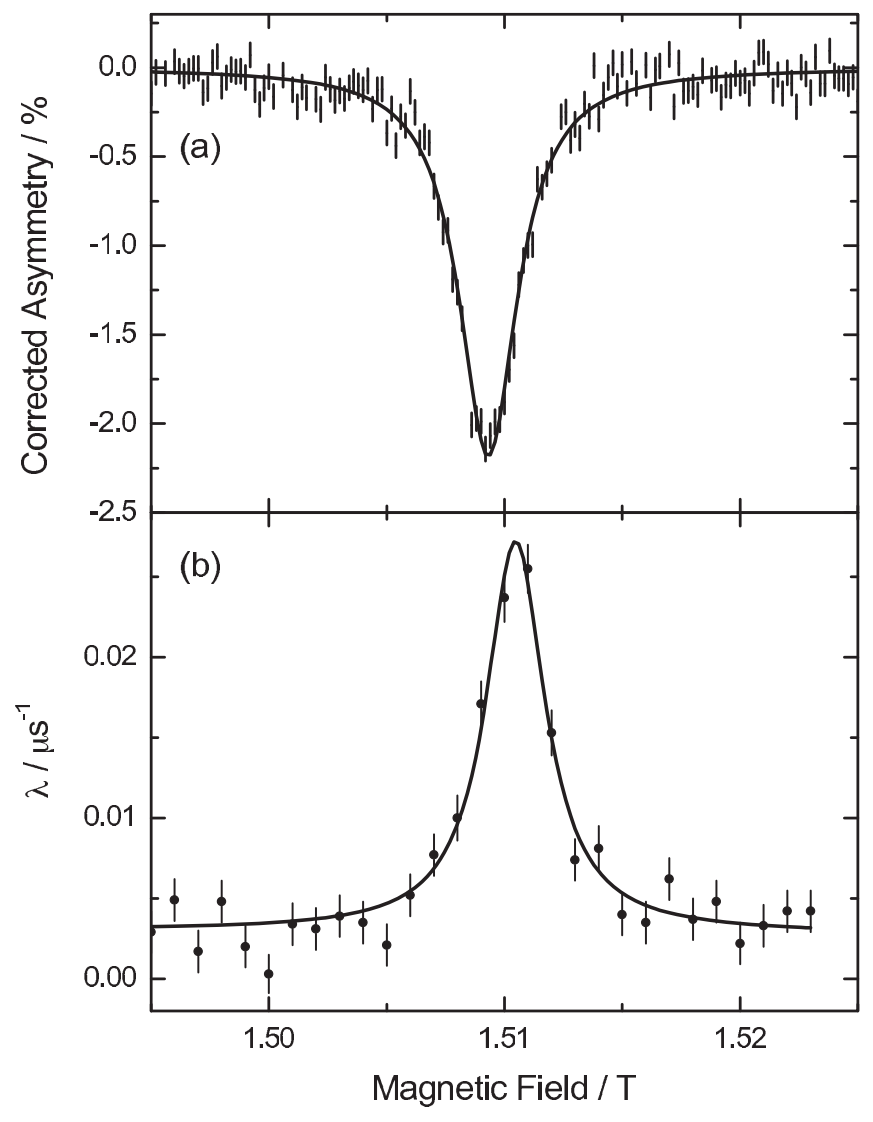

FIG. 10. The $|\Delta M|=1$ resonance of the exo Mu adduct of norbornene in (a) time-integral mode, $286 \mathrm{~K}$ and (b) time-differential mode, $282 \mathrm{~K}$. The shift is due to the temperature dependence of the hfcc.

mask could short-circuit the coil or provide an easy path for breakdown currents.

We measured the semiconductor $\mathrm{CdS}$, where the muon acts as a donor impurity and binds an electron in a shallow state at low temperature. ${ }^{22}$ The low hyperfine constant $A<$ $1 \mathrm{MHz}$ gives a spectrum with two sidebands (electron spin up

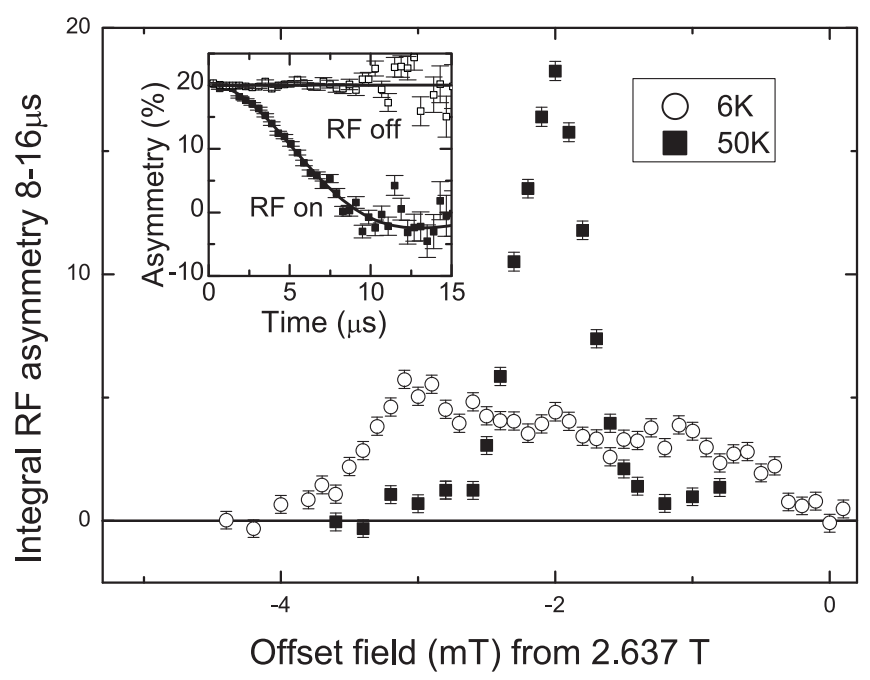

FIG. 11. Resonance lines in CdS below $(6 \mathrm{~K})$ and above $(50 \mathrm{~K})$ the shallow donor ionisation temperature. Working frequency $373 \mathrm{MHz}$. Inset: time domain data on-resonance at $50 \mathrm{~K}$. or down) close to the diamagnetic line. The reason for a high field measurement is that the electrons may show an average spin polarisation, depending on $B / T .^{23}$

We used fixed tuning capacitors determining the RF frequency, so the main field was set close to the resonant position and was put in a persistent state, and the $z$ coil was used to scan through the lines, over a range of $5 \mathrm{mT}$. Taking data with the RF field alternately on and off allows us to cancel any background relaxation.

Figure 11 shows the results, plotted as the RF on/off difference of integral asymmetry. At $50 \mathrm{~K}$, the time domain data can be fitted to give $B_{1} \approx 0.3 \mathrm{mT}$, and the width of the Lorentzian line profile in the integral data is consistent with this.

\section{CONCLUSIONS}

A new state-of-the-art high field muon spectrometer, optimised for a pulsed muon source, has been successfully constructed and commissioned at the ISIS pulsed neutron and muon source. The instrument is particularly suited to time-differential muon measurements at high fields, including measurements which exploit level-crossing or radiofrequency resonance techniques. The challenges to the construction of the instrument-including stringent requirements on the instrument magnet, systems for compensating for remanent fields, and methods for aligning the beam with the magnetic field axis-have been overcome. A detector array working over the full field range has been constructed which provides high time-differential data rates, minimises double counting, and provides stable operation over long time periods allowing field-dependent backgrounds to be corrected.

The instrument measured its first muon spectrum in a field of $5 \mathrm{~T}$ at 14:54 on September 18, 2009. Demonstration experiments have shown the instrument capable of meeting all the original scientific aims that formed the raison-d'etre for its construction. The instrument is now in routine operation as a part of the ISIS pulsed muon facility.

\section{ACKNOWLEDGMENTS}

The construction of HiFi was funded by a Facility Development grant awarded by the Science and Technology Facilities Council (Grant reference No. FDPG/082). Much of the detector design work was supported by the European Commission under the 6th Framework Programme through the Key Action: Strengthening the European Research Area, Research Infrastructures: Contract No: RII3-CT-2003-505925. Instrument optimisation was supported by the European Commission under the 7th Framework Programme through the Key Action: Strengthening the European Research Area, Research Infrastructures. Contract No: CP-CSA_INFRA-2008-1.1.1 Number 226507-NMI3. We acknowledge the assistance of a large number of the ISIS technical and support staff involved in its construction. We thank D. Prabhakaran for the $\mathrm{Ca}_{3} \mathrm{Co}_{2} \mathrm{O}_{6}$ sample used in Sec. VIII A. 
${ }^{1}$ A. Schenck, Muon Spin Rotation Spectroscopy (Hilger, Bristol, 1985).

${ }^{2}$ S. J. Blundell, Contemp. Phys. 40, 175 (1999).

${ }^{3}$ Muon Science: Muons in Physics, Chemistry, and Solids, in Proceedings of the 50th Scottish University Summer School in Physics, A Nato Advanced Study Institute, edited by S. L. Lee, S. H. Kilcoyne, and R. Cywinski (IOP Publishing, Bristol, 1999), Vol. 51.

${ }^{4}$ G. H. Eaton, M. A. Clarke-Gayther, C. A. Scott, C. N. Uden, and W. G. Williams, Nucl. Instrum. Methods Phys. Res. A 342, 319 (1994).

${ }^{5} \mathrm{See}$ http://lmu.web.psi.ch/facilities/facility.html for high field instruments LTF and ALC at PSI.

${ }^{6} \mathrm{See}$ http://cmms.triumf.ca/equip/spect.html for high field instruments HITIME, HELIOS and DR at TRIUMF.

${ }^{7}$ K. Nishiyama, T. Azuma, K. Ishida, T. Matsuzaki, J. Imazoto, T. Yamazaki, and K. Nagamine, Hyperfine Interact. 32, 887 (1986).

${ }^{8}$ P. J. C. King, S. P. Cottrell, S. F. J. Cox, G. H. Eaton, A. D. Hillier, J. S. Lord, F. L. Pratt, T. Lancaster, and S. J. Blundell, Physica B 326, 260 (2003).

${ }^{9}$ Z. Salman, P. J. Baker, S. J. Blundell, S. P. Cottrell, S. R. Giblin, A. D. Hillier, B. H. Holsman, P. J. C. King, T. Lancaster, J. S. Lord, I. McKenzie, J. Nightingale, F. L. Pratt, and R. Scheuermann, Physica B 404, 978 (2009).

${ }^{10}$ C. Wang and P. Gifford, Cryocoolers 11, 205 (2002).

${ }^{11}$ G. H. Eaton, C. A. Scott, and W. G. Williams, in Proceedings of the International Workshop on Low Energy Muon Science, Santa Fe (LEMS 93), (Los Alamos National Laboratory, New Mexico, 1994).
${ }^{12}$ M. C. Lynch, S. P. Cottrell, P. J. C. King, and G. H. Eaton, Physica B 326, 270 (2003).

${ }^{13}$ T. Lancaster, T. Shiroka, R. Scheuermann, A. Stoykov, Z. Salman, S. P. Cottrell, P. J.C. King, J. S. Lord, and F. L. Pratt, Nucl. Instrum. Methods Phys. Res. A 580, 1578 (2007).

${ }^{14}$ U. Rohrer, "PSI Graphic Turtle Framework," Based on a CERN-SLACFERMILAB version by K. L. Brown et al.

${ }^{15}$ G. Majer, R. Messer, A. Seeger, W. Templ, K. Fürderer, M. Gladisch, and D. Herlach, Philos. Mag. Lett. 57, 57 (1988).

${ }^{16}$ V. F. Hardy, M. R. Lees, O. A. Petrenko, D. M. Paul, D. Flahaut, S. Hébert, and A. Maignan, Phys. Rev. B 70, 064424 (2004).

${ }^{17}$ S. Takeshita, J. Arai, T. Goko, K. Nishiyama, and K. Nagamine, J. Phys. Soc. Jpn. 75, 034712 (2006).

${ }^{18}$ C. P. Slichter, Principles of Magnetic Resonance, 3rd ed. (Springer-Verlag, New York, 1996).

${ }^{19}$ E. Roduner, Chem. Soc. Rev. 22, 337 (1993).

${ }^{20}$ I. McKenzie and E. Roduner, Naturwiss. 96, 873 (2009).

${ }^{21}$ M. Ricco, R. de Renzi, and E. Roduner, Phys. Lett. A 129, 390 (1988).

${ }^{22}$ J. M. Gil, H. V. Alberto, R. C. Vilão, J. P. Duarte, P. J. Mendes, L. P. Ferreira, N. Ayres de Campos, A. Weidinger, J. Krauser, C. Niedermayer, and S. F. J. Cox, Phys. Rev. Lett. 83, 5294 (1999).

${ }^{23}$ H. V. Alberto, A. Weidinger, R. C. Vilão, J. Piroto Duarte, J. M. Gil, J. S. Lord, and S. F. J. Cox, Phys. Rev. B 81, 245205 (2010). 